\title{
Visualization and Quantitation of Immobilized Biopolymer Molecules During the Steps of Microarray Production and Microarray Reading
}

\author{
V.V. Golovlev,* M.P. McCann,* L.F. Allard** and H.M. Meyer** \\ * Sci-Tec, Inc., 10425 Cogdill Rd., \#300, Knoxville, TN 37932-3391 \\ ** Oak Ridge National Laboratory, PO Box 2008, Oak Ridge, TN 37831-6064
}

Production of microarrays and high multiplexity bioassays often require chemical modification of polymer surfaces on which probe biomolecules will be immobilized [1,2]. Manufacturing microarrays requires monitoring surface modifications during steps of activation, array printing, surface blocking, analyte binding, and on the final step of reading hybridized microarrys. We present here a new technique of using nanometer-size gold particles for characterization of the binding properties of microarray substrates, and for quantitative measurement and two-dimensional mapping density of DNAs and proteins. The approach is based on non-specific electrostatic binding of gold particles to biopolymer molecules, and is different from the conventional approach of using gold conjugates for specific binding/hybridization to molecular targets $[3,4]$. Figure 1a shows an example of non-specific binding of $250 \mathrm{~nm}$ gold particles to a spot covered by a monolayer of positively charged poly-L-lysine molecules.

Biopolymers, such as DNA and proteins, have different affinity to colloidal gold as a result of the net electric charge carried by the molecules. When immobilized on a surface, such biopolymers increase or decrease the capacity of the surface to bind gold particles. For visualization of biopolymer molecules by non-specific labeling, the property of the entire substrate surface needs be specially engineered. In this study we have adjusted the affinity of the surface to bind gold nanoparticles by linking up thiol groups through mercaptosilane-based surface modification chemistry. A Hitachi S-4700 SEM and a Physical Electronics PHI-680 scanning Auger nanoprobe were used to characterize nanoparticle distribution and to verify polymer surface modification. The presence and density of thiol groups on the polymer surface were confirmed by studying the Auger spectra of the chemically modified microarray surface. Figure 2 shows an example of the AES spectra acquired for (A) mercaptosilane-modified and (B) non-modified polymer surfaces. The presence of the sulfur peak in the spectrum of the modified substrate indicates attachment of thiol groups to the surface. Thiol groups increased affinity of the surface for gold nanoparticles and also provided binding groups for immobilization of proteins and chemically modified polinucleotides. Different regimes of binding of colloidal gold to the surface were observed vs. ionic strength of the developing colloidal solution, as was shown via SEM imaging. When microarrays are developed in solution with low ionic strength (less than $50 \mathrm{mM}$ of $\mathrm{NaCl}$ ), the electrostatic particle-particle interaction plays the dominant role. The charge causes repulsion of particles, which yields a relatively low coverage of the surface by gold particles, as illustrated in Fig. 1b. When a developing solution of higher ionic strength is used $(100 \mathrm{mM}$ or higher of $\mathrm{NaCl})$, the charge of the nanoparticles is neutralized and clustering of the bound particles is often observed, as shown in Fig. 1c.

The SEM and SAN were used to optimize protocols of the labeling technique, which then was used for monitoring the process of microarray manufacturing and for detection and quantification of DNA and proteins in microarray experiments. The method of non-specific binding for detection of biopolymers is sensitive and easy to implement. By immobilizing different quantities of 
DNA and proteins we found that typically less than $50 \mathrm{pg}$ of DNA and less than $20 \mathrm{pg}$ of protein can be detected. In this labeling method no chemical modification of probe and target molecules for detection is required, which uniquely position this detection method vs. the conventional fluorescence detection of biopolymers.

\section{References}

[1] Hegde, P. et al., BioTechniques 29, 549-562 (2000).

[2] M.F. Templin et al., TRENDS in Biotechnology, 20(4), 160-166 (2002)

[3] Taton, T.A., Mirkin, C.A., Letsinger, R.L., Science 289, 1757-1760 (2000).

[4] G. Morel, A. Cavalier, CRC Press, 2001

[5] Research sponsored in part by the Asst. Sec. for Energy Efficiency and Renewable Energy, Office of FreedomCAR and Vehicle Technologies, as part of the High Temperature Materials Laboratory User Program, Oak Ridge National Laboratory, managed by UT-Battelle LLC for the U.S. DOE, and by research contract N43-ES-25492 from National Institute of Environmental Health Sciences.
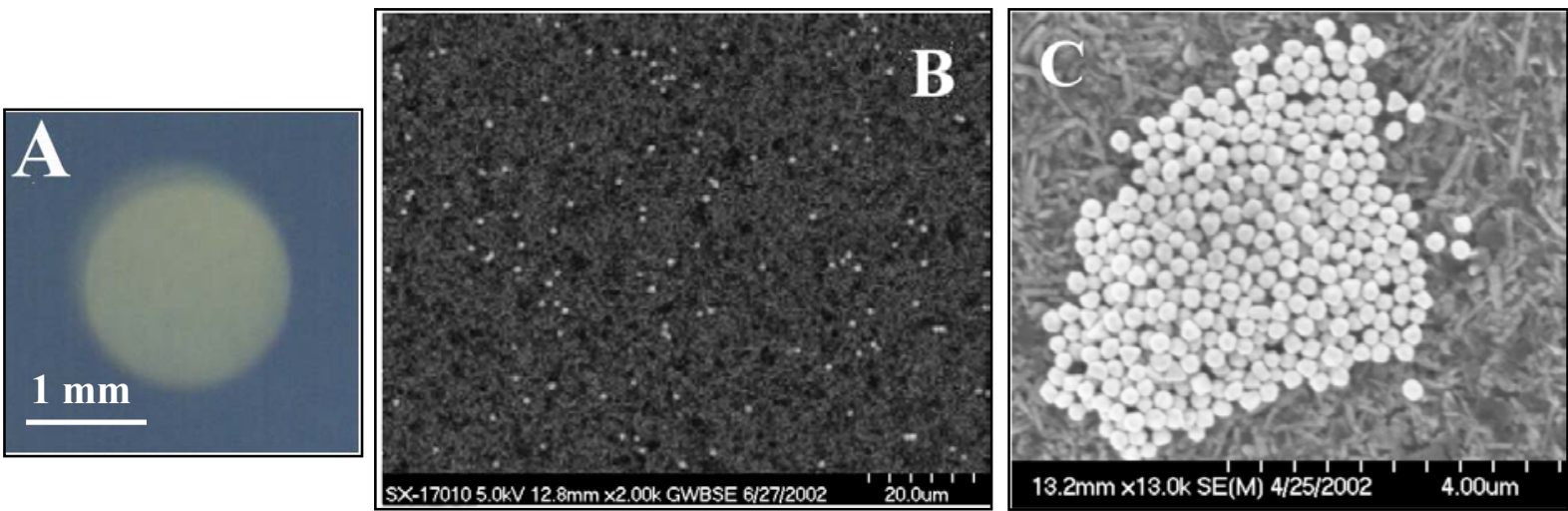

FIG. 1. (a) Binding of 250-nm gold particles by poly-L-lysine molecules immobilized on polymer substrate. (b) SEM photographs captured when the spot was developed in a buffer, which has low ionic strength $(<50 \mathrm{mM} \mathrm{NaCl})$; (c) is example of formation of clusters of gold nanoparticles on substrate developed in high ionic strength buffer $(>100 \mathrm{mM} \mathrm{NaCl})$.

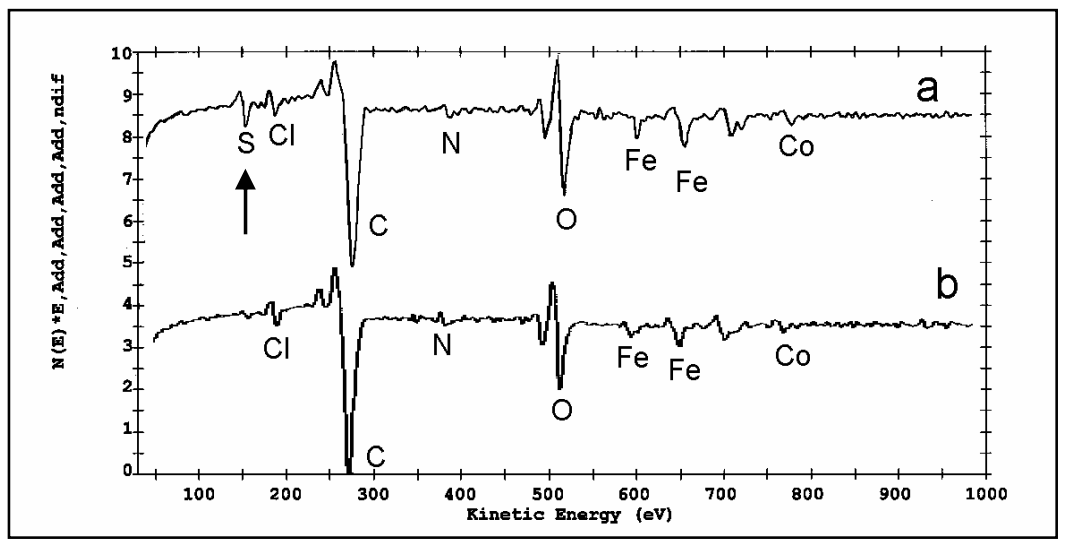

FIG. 2. Auger spectrum for (a) a mercaptosilane-treated and (b) a non-treated areas of the polymer surface covered by a thin magnetizable layer. 